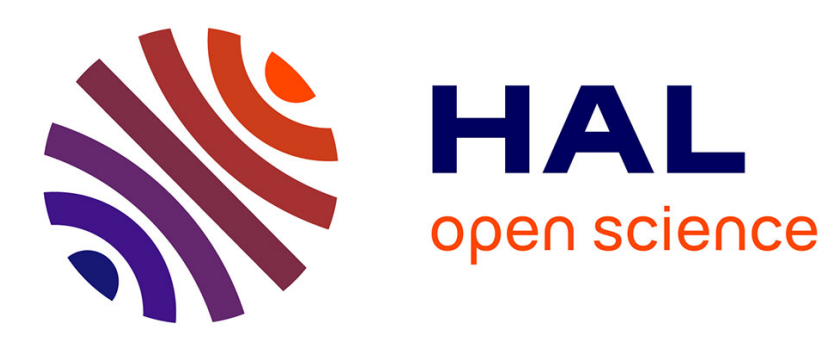

\title{
QUANTITATIVE APPROACHES FOR THE STUDY OF MICROTUBULE ASTER MOTION IN LARGE EGGS
}

\author{
Hirokazu Tanimoto, Nicolas Minc
}

\section{- To cite this version:}

Hirokazu Tanimoto, Nicolas Minc. QUANTITATIVE APPROACHES FOR THE STUDY OF MICROTUBUlE ASTER MOTION IN LARGE EGGS. Thomas Lecuit. Cell Polarity and Morphogenesis Edited by Thomas Lecuit, 2017. hal-01864175

\section{HAL Id: hal-01864175 \\ https://hal.science/hal-01864175}

Submitted on 29 Aug 2018

HAL is a multi-disciplinary open access archive for the deposit and dissemination of scientific research documents, whether they are published or not. The documents may come from teaching and research institutions in France or abroad, or from public or private research centers.
L'archive ouverte pluridisciplinaire HAL, est destinée au dépôt et à la diffusion de documents scientifiques de niveau recherche, publiés ou non, émanant des établissements d'enseignement et de recherche français ou étrangers, des laboratoires publics ou privés. 


\title{
QUANTITATIVE APPROACHES FOR THE STUDY OF MICROTUBULE
}

\author{
ASTER MOTION IN LARGE EGGS
}

Hirokazu Tanimoto and Nicolas Minc*

Institut Jacques Monod, CNRS UMR 7592, 15 rue Hélène Brion, 75205 Paris Cedex 13, FRANCE

*Correspondence: nicolas.minc@ijm.fr

Keywords: Microtubule aster; Dynein; Sea urchin; 3D tracking, Laser ablation 


\begin{abstract}
:
The proper positioning of microtubule (MT) asters underlies fundamental processes such as nuclear centration, cell polarity, division positioning and embryogenesis. In large eggs and early blastomeres, MT asters may exhibit long range motions with atypical speed and precision, to target their functional position. The biophysical mechanisms regulating such motions remain however largely unknown. The centration of sperm asters in sea urchin embryos is a stereotypical example of such aster long range motion. In this chapter, we describe methods developed in this system to (i) quantify sperm aster 3D motion with confocal microscopy and automated image analysis, and (ii) severe a portion of astral MTs with a UV laser. These methods may serve as a template to dissect the generic mechanisms of aster motion and force production in other embryos and cell types.
\end{abstract}




\section{Introduction}

The spatio-temporal control of microtubule (MT) aster position in the cell is crucial to various biological contexts and has received extensive attention in the context of cell division plane specification (Elric \& Etienne-Manneville, 2014; K. Kimura \& Kimura, 2014; Kiyomitsu, 2014; McNally, 2013; Minc \& Piel, 2012). To precisely control daughter cell size and shape, cells may sense their shape to place the division plane accordingly. MT asters, which are star-shaped arrays of MTs radiating from centrosomes, may serve to convert cell shape information to division plane positioning. In small cells such as adherent animal cells, metaphase asters around the spindle attach to cell cortex and interact with molecular motors such as dynein at the cortex to mediate spindle and consequent division-plane positions (Kiyomitsu \& Cheeseman, 2013). In contrast, in large cells such as echinoderm, amphibian, and fish eggs and early blastomeres, metaphase asters are too small to reach the cell cortex (Mitchison et al., 2012). In these cells, interphase/telophase asters fill large portion of the cytoplasm and have been proposed to mediate division plane determination through dynein pulling forces produced along MTs in bulk cytoplasm (Minc, Burgess, \& Chang, 2011; Tanimoto, Kimura, \& Minc, 2016; Wuhr, Tan, Parker, Detrich, \& Mitchison, 2010).

The functional positioning of MT asters relies on precise aster motion. While many studies have focused on the mechanisms that determine aster final position, little is known about how asters move inside cells. This question becomes particularly prominent in the context of eggs and early blastomeres given their large size and the rapidity of early embryonic cell cycles. A typical example, conserved in most species, is sperm aster centration. After fertilization sperms bring centrioles inside eggs to nucleate a sperm aster that moves to the exact center of the large egg 
(Chambers, 1939; Hamaguchi \& Hiramoto, 1980). These asters continuously grow in size and move at speeds of several microns per minute to reach the exact center of these large cells. The lack of information on sperm aster motion, may be in part associated to difficulties of imaging motion in 3D, in large cells, and on the limited set of quantitative methodologies to manipulate aster forces in space and time. Recently, we developed quantitative methods, based on 3D microscopy and laser ablation to address the biophysical mechanisms driving sperm aster motion in sea urchin embryos (Tanimoto, et al., 2016). The first method uses confocal microscopy and MATLAB-based automated image analysis to precisely track aster motion in 3D, for normal spherical eggs and for eggs shaped in microchambers. This method serves to obtain motion characteristics such as travelling distance, speed, and velocity orientation. The second method uses a UV laser ablation setup to severe astral MTs in different positions and monitor the consequence on speeds and trajectories. Since the presented methods do not rely on any peculiar features of the organism, we emphasize they could be useful to study aster motion questions in other systems. 


\section{Three-dimensional tracking of microtubule asters in sea urchin embryos}

This section describes a basic method to track aster centration in $3 \mathrm{D}$ with high precision. The method includes cell handling, confocal microscopy, PDMS chamber operation, and image analysis using a custom-made code written in MATLAB.

\subsection{Detecting 3D aster position using confocal microscopy}

\section{$\underline{\text { Materials }}$}

Sea urchin (Paracentrotus lividus), Sterile water, Artificial sea water (ASW; Instant Ocean; Reef Crystals), $80 \mu \mathrm{m}$ Nitex mesh (Sefar), Hoechst 33342 (Sigma), Glass bottom dish (50 mm diameter, thickness no. 1.5; MatTek Corporation), Protamine sulfate (Sigma), PDMS chamber (prepared as described in (Chang, Atilgan, Burgess, \& Minc, 2014)), Plasma cleaner (Harrick Plasma), Air gun, Spinning disk confocal microscopy (Nikon Ti-Eclipse combined with Yokogawa CSU-X1 spinning-head and Hamamatsu EM-CCD camera) equipped with a 40x objective (Nikon Plan Fluor, NA 1.3).

\section{$\underline{\text { 2.1.1 Sample preparation }}$}

a. Preparation of imaging dishes. Treat glass-bottom dishes with $1 \%$ protamine sulfate for 2 min. Rinse the dishes thoroughly with sterile water and dry them under the hood. This treatment makes the dishes positively charged, on which eggs will adhere. The coated dishes can be stored in a clean box at room temperature for at least 1 month. 
b. Obtain gametes from adult sea urchins by intracoelomic injection of $0.5 \mathrm{M} \mathrm{KCl}$. Keep sperm at 4 degree and use them within $\sim 1$ week. Rinse the eggs twice using ASW and store them at 16 degree. Eggs should be used within the day of collection.

c. Remove the jelly coat of unfertilized eggs to facilitate cell adhesion by passing them three times though an $80-\mu \mathrm{m}$ Nitex mesh. Alternatively, acidic treatment may also be used (1 2 min treatment with pH 5.5 ASW at 16 degree, see (Cheers \& Ettensohn, 2004) for details).

d. Place a $6 \mathrm{~mL}$ solution of de-jellied eggs on a coated glass-bottom dish with adequate dilution in ASW. Add the DNA dye Hoechst 33342 at a final concentration of $1 \mu \mathrm{g} / \mathrm{mL}$. If the Hoechst signal intensity of the male pronucleus is too weak, one can increase Hoechst concentration up to $5 \mu \mathrm{g} / \mathrm{mL}$, which does not alter aster centration dynamics in our experimental condition.

\section{$\underline{\text { 2.1.2 Imaging }}$}

$\underline{\text { Setup }}$

The position of MT asters is determined by tracking the center of the male pronucleus bound to centrosomes which act as microtubule organizing centers. The male pronucleus is visualized with the fluorescent DNA dye Hoechst, and its position is measured by taking confocal timelapse $\mathrm{Z}$ stacks (Fig. 1 A). The interval of the $\mathrm{Z}$ slice should be small enough to precisely determine the position of the aster in $\mathrm{Z}$. However, a too small slice interval reduces the temporal resolution and can cause photo-toxicity. A Z-interval of $3 \mu \mathrm{m}$ is sufficient to catch the male pronucleus ( $2 \mu \mathrm{m}$ size) in a single focal plane in one of the slice of the stack (Fig. $1 \mathrm{~B})$. The number of $\mathrm{Z}$ slices is set so that the confocal stack covers a hemisphere of the eggs which allows to monitor the whole centration. 15 slices are sufficient to cover the hemisphere of a sea urchin egg (90 $\mu \mathrm{m}$ in diameter). The source laser is a $405 \mathrm{~nm}$ laser. Laser intensity and exposure time 
are adjusted to minimize photo-toxicity. It takes $\sim 8 \mathrm{sec}$ to take one $\mathrm{Z}$ stack in our setup and the temporal interval, $\Delta \mathrm{t}$ is set to $10 \mathrm{sec}$.

\section{Procedure}

a. Place the sample on the microscope stage.

b. Dilute dry sperm 1000-fold in ASW by putting $4 \mu \mathrm{L}$ sperm into $4 \mathrm{~mL}$ ASW. Activate them by vigorous aeration. Add the sperm solution dropwise to the dish under the microscope.

c. Detect the $\mathrm{Z}$ position of a sperm entering an egg. Choose the initial $\mathrm{Z}$ position of the timelapse movie so that the $\mathrm{Z}$ stack volume includes both initial male pronucleus position and the egg center.

d. Start time-lapse acquisition. The entire aster centration takes $\sim 10-15 \mathrm{~min}$ in normal conditions (Fig. 1 C).

\subsubsection{Imaging in PDMS chamber}

The 3D tracking framework described in the last section can also be used to monitor aster motion in eggs shaped into PDMS chambers. This subsection describes additional procedures required for imaging aster motion in this condition.

\section{$\underline{\text { Procedure }}$}

a. Prepare appropriate PDMS chambers with cell-size microwells (see (Chang, et al., 2014) for detailed protocols for chamber design and fabrication). 
b. Clean PDMS chambers and glass cover slips. The cover slips should be larger than the PDMS slabs. Treat them with Acetone, Isopropanol and then rinse with sterile water. Dry them thoroughly using an air gun and keep them on kimwipes.

c. Activate PDMS chambers, with micro-wells facing up with a plasma cleaner. Check if they become hydrophilic by putting a drop of ASW. If not, dry them and repeat plasma activation possibly by increasing treatment time. Keep activated chambers in ASW until use. The activation lasts $\sim 4$ hours.

d. Go to the microscope. Fertilize eggs in $15 \mathrm{~mL}$ falcon tubes. Wait $\sim 1$ min until the eggs accumulate at the bottom of the falcon tube by sedimentation. Place $40 \mu \mathrm{L}$ of a dense egg solution on the clean coverslip.

e. Remove ASW from the activated PDMS slab with a kimwipe. Slowly apply the PDMS slab on the top of the egg solution with microwells facing down. Do not press the slab.

f. Remove excess ASW from the slab side using kimwipe. The slab approaches to the coverslip which causes eggs to go into the microwells.

g. Place the sample under the microscope. Find a cell successfully shaped in a well and acquire time-lapse images as described in 2.1.1 (Fig. 1 G). It typically takes 3-5 min from sperm addition to the beginning of the image acquisition (steps d-g).

\subsection{Quantification of aster 3D motion}

\section{$\underline{\text { Materials }}$}

Standard desktop PC with a MATLAB software (supplemented with the statistical and image analysis toolboxes; Mathworks) and dedicated scripts (available upon demand). 


\section{Image analysis framework}

The 3D position of the male pronucleus is determined from a fluorescence time-lapse $\mathrm{Z}$ stack of images using home-built MATLAB scripts. Aster $Z$ position is determined by detecting the plane in which the male pronucleus is in focus (Fig. $1 \mathrm{~A}$ and B). Aster XY position is then determined by detecting the $2 \mathrm{D}$ center of male pronucleus within the focal plane. The method enables a precise positional detection: the precision of the position in $\mathrm{XY}$ is estimated to be below $1 \mu \mathrm{m}$ (using immobile sperm on the dish), and that in $\mathrm{Z}$ to be $\sim 3 \mu \mathrm{m}$ (set by the $\mathrm{Z}$ slice interval) (Fig. $1 \mathrm{C})$.

The aster 3D position $\vec{X}(t)$ is used to extract aster motion characteristics (Fig. 1 D-F). Aster travelling distance $L(t)$ is defined as the distance between aster initial and current positions $L(t)=|\vec{X}(t)-\vec{X}(0)|$. Aster instantaneous velocity is defined as $\vec{V}(t)=$ $\{\vec{X}(t+\tau)-\vec{X}(t)\} / \tau$, where $\tau=30$ sec, and $|V|$ is the aster speed. Aster 3D orientation is defined using spherical coordinate;

$\vec{V}=\left(\begin{array}{c}V_{x} \\ V_{y} \\ V_{z}\end{array}\right)=\left(\begin{array}{c}|V| \sin \theta \cos \varphi \\ |V| \sin \theta \sin \varphi \\ |V| \cos \theta\end{array}\right)$,

where $\theta$ and $\varphi$ are polar and azimuthal angles, respectively.

Since aster motion involves fluctuations around a persistent directed motion, it may be non-trivial to define a travelling distance for asters undergoing a non-straight motion. In the case of sperm asters' motion in shape-manipulated cells, aster rotations are very sharp so the migrating path consists of multiple straight lines and small part connecting them (Fig. $1 \mathrm{G})$. The travelling distance of these asters can be defined as a summation of the passed paths and the distance from the last rotating position. 


\section{$\underline{\text { Procedure }}$}

a. Open MATLAB and run the script.

b. Choose the $\mathrm{Z}$ slice in which the male pronucleus is in focus at the first time point.

c. The script opens the image of the first time point. Select manually a rough position of the male pronucleus in the image.

d. The script updates the pronucleus position in the first time point by detecting the male pronucleus shape based on thresholding.

e. The scripts automatically find the $\mathrm{Z}$ plane in which the male pronucleus is in focus at successive time points, and detect the XY position in the plane.

f. The script allows to check the tracking results in an interactive way. The script generates images of detected outline of male pronucleus superimposed on the real image of the male pronucleus at the focal plane. The tracking results are checked manually based on these images. If an error is found at a given time point, the aster position at this point may be updated by changing the initial position estimation and/or the threshold value for male pronucleus shape detection.

\section{$\underline{\text { Output }}$}

The scripts generate an aster 3D trajectory (Fig. $1 \mathrm{C}$ ) and computes aster motion characteristics such as travelling distance (Fig. 1 D) and amplitude and orientation of the instantaneous velocity (Fig. $1 \mathrm{E}$ and F). These outputs are saved both as plot figures and as MatLab files which can be exported to excel. These quantifications can be used to characterize complicated aster motion such as rotation(s) in shape-manipulated cells (Fig. $1 \mathrm{G}-\mathrm{K})$. 


\subsection{Laser ablation of astral microtubules and in-situ immunostaining}

This section describes: (i) a method to photo-ablate MTs on different parts of migrating sperm asters, and (ii) a method to perform rapid in-situ immunostaining of ablated asters.

\section{$\underline{\text { Materials }}$}

In addition to the materials required for tracking: the confocal microscopy is equipped with a high-power pulsed 355-nm UV laser system (iPulse, Roper Technologies) operated by iLas2 software (Roper Technologies). Chemicals for immunostaining, as listed in (Minc, et al., 2011).

\section{$\underline{\text { 3.1.1 Laser ablation of astral microtubules }}$}

\section{$\underline{\text { Procedure }}$}

a. Prepare a sample and place it on the microscope as described in 2.1.1.

b. Fertilize eggs under the microscope by adding activated sperm.

c. Select an egg in which the sperm enters close to the equatorial plane so that the aster moves mostly in plane.

d. Start a time-lapse acquisition to monitor the aster motion as described in 2.1.2.

Since the aster moves almost in-plane, a $\mathrm{Z}$ stack is not always necessary for the measurement. We often reduce the number of $\mathrm{Z}$ slice to 5 and $\Delta \mathrm{Z}$ to $1 \sim 2 \mu \mathrm{m}$.

e. Perform laser ablation when the aster has moved $\sim 25 \mu \mathrm{m}$ away from the cortex.

The size of the target aster is a critical parameter to obtain a significant and reproducible effect of ablation on aster motion. The ablation position should be far enough from both the aster center and cell cortex. If the ablation position is too close to the male pronucleus, the laser irradiation bleaches the Hoechst signal and prevents subsequent tracking of the male pronucleus. 
In addition, ablating too close to the aster center oftentimes stops aster motion for unknown reason(s). If the ablation position is too close to the cell cortex, the irradiation affects cell integrity and oftentimes leads to cell bursting.

We perform laser ablation when the aster has moved $\sim 25 \mu \mathrm{m}$ from the cortex, when the aster size is around $50 \mu \mathrm{m}$ (Tanimoto, et al., 2016). The irradiated line is set to be $\sim 30 \mu \mathrm{m}$ long. The irradiated position is set to be $10 \sim 15 \mu \mathrm{m}$ away from the aster center (Fig. 2 A). This setup enables us to perform laser ablation either at the front or on the side of MT asters. However, this setup does not work for rear MT ablation as the laser line tends to cross the cell cortex, and yields to cell bursting typically 1 min after ablation.

The laser power is set to be $50 \%$. Each irradiated line consists of three 350 -nm beam lines spaced by an interval of $1 \mu \mathrm{m}$ (by setting the "line thickness" parameter in the iLas2 software to be 2 in the setting for the 40x objective). Each line irradiation takes $5-7 \mathrm{~ms}$ and is iterated 400 times. To ablate the MT aster in 3D, the ablation is performed at three different heights: at the aster center and $5 \mu \mathrm{m}$ above and below. The total time needed to complete the ablation is $\sim 10 \mathrm{sec}$, thus if the experiments do not need fine temporal resolution, ablations of several eggs may even be performed in parallel using a motorized XY stage. This setup is sufficient to significantly reduce MT length and density at the ablated region (Fig. 2 B).

f. Continue time-lapse acquisition (Fig. 2 C). Analyze aster motion as described in 2.2 to quantify the effects of ablation assay.

Laser ablation may sometimes causes small egg movement. In these cases, we determine the cell movement from the auto-fluorescence of the cytoplasm or DIC and subtract it from the aster motion to get the aster motion in the cell referential frame. 


\section{$\underline{\text { Output }}$}

The laser ablation assay of astral MTs serves to dissect the direction of MT forces that drives aster motion. In the case of sperm asters of sea urchin eggs, side ablation shifts the aster centration path away from the ablation side (Fig. 2 D and E), and front ablation leads to transient reverse of aster velocity orientation (Tanimoto, et al., 2016); both suggesting that aster motion is driven by pulling forces mediated by MTs.

\section{$\underline{\text { 3.1.2 In-situ immunostaining of ablated asters }}$}

\section{$\underline{\text { Procedure }}$}

a. Prepare PDMS slabs with a wide and straight perfusion channel and two inlet holes. The dimension of the channel is $8 \mathrm{~mm} * 25 \mathrm{~mm}$ and $70 \sim 75 \mu \mathrm{m}$ in height. The height is $\sim 10 \%$ smaller than the egg diameter to slightly flatten the eggs. The slight flattening stabilizes cell position and forces sperms to enter around the cell equatorial plane. Pierce holes at the two extremes of the channel to allow liquid perfusion.

b. Choose coverslips larger than the slab dimension. Clean the PDMS slabs as well as coverslips using acetone and isopropanol. Rinse them with water and thoroughly dry with an air gun.

c. Place a $25 \mu \mathrm{L}$ solution of unfertilized egg onto the PDMS channel facing up. Put the coverslip onto the PDMS slab, and push onto the periphery to seal the channel.

d. Fertilize the eggs in the chamber by introducing activated sperm from the channel inlet hole.

e. Perform laser ablation as described in 3.1.1. 
f. Perform fixation immediately after laser ablation, by rapidly introducing the fixative from the hole, following a fixation procedure described in (Minc, et al., 2011). With this setup one can fix samples within less than $10 \mathrm{sec}$ after ablation.

g. Perform successive steps of immunostaining by exchanging solutions under the microscope, to ensure that the eggs do not move nor deform during the process.

\section{$\underline{\text { Output }}$}

Since the re-growth of ablated astral MTs may be very quick (Hyman, 1989), visualizing the effects of laser ablation requires rapid in-situ immunostaining. The presented protocol enables rapid in-situ immunostaining of ablated MT asters that is crucial to assess the effects of laser ablation on aster size and density (Fig. 2 B).

\section{Conclusions}

The methods described in this chapter serve to study aster motion in sea urchin embryos and possibly for other species for which characterization of aster motion remains to be analyzed and understood (Stewart-Savage \& Grey, 1982). The dynamics of aster motion reflects the underlying mechanisms of aster force production (A. Kimura \& Onami, 2005, 2007). However, our studies suggest that MT asters in different model organisms could exhibit qualitatively different motions even if they may rely on common biological principles (Tanimoto, et al., 2016). These observations should stimulate the developments of unified biophysical theories, which will likely require further quantitative experiments of aster motion in various cell types. Laser ablation is a powerful tool to dissect the direction of MT forces, and may serve to obtain 
more quantitative information on the functional form of aster force dependency on aster size and shape; which are key for constructing quantitative mathematical models.

\section{Acknowledgments:}

We thank Akatsuki Kimura, François Rousset, and members of the Minc Laboratory for technical help and discussion. This work is supported by the CNRS and grants from the "Mairie de Paris emergence" program, the FRM "amorçage" grant AJE20130426890 and the ERC (CoG Forcaster $\left.\mathrm{N}^{\circ} 647073\right)$. 


\section{References:}

Chambers, E. L. (1939). The movement of the egg nucleus in relation to the sperm aster in the echinoderm egg. Journal of Experimental Biology, 16(4), 409-424.

Chang, F., Atilgan, E., Burgess, D., \& Minc, N. (2014). Manipulating Cell Shape by Placing Cells into Micro-fabricated Chambers. Mitosis: Methods and Protocols, 281-290.

Cheers, M. S., \& Ettensohn, C. A. (2004). Rapid microinjection of fertilized eggs. Methods Cell Biol, 74, 287-310.

Elric, J., \& Etienne-Manneville, S. (2014). Centrosome positioning in polarized cells: common themes and variations. Exp Cell Res, 328(2), 240-248.

Hamaguchi, M., \& Hiramoto, Y. (1980). Fertilization Process in the Heart Urchin, Clypeaster Japonicus Observed with a Differential Interference Microscope. Development, growth \& differentiation, 22(3), 517-530.

Hyman, A. A. (1989). Centrosome movement in the early divisions of Caenorhabditis elegans: a cortical site determining centrosome position. J Cell Biol, 109(3), 1185-1193.

Kimura, A., \& Onami, S. (2005). Computer simulations and image processing reveal lengthdependent pulling force as the primary mechanism for $\mathrm{C}$. elegans male pronuclear migration. Dev Cell, 8(5), 765-775.

Kimura, A., \& Onami, S. (2007). Local cortical pulling-force repression switches centrosomal centration and posterior displacement in C. elegans. J Cell Biol, 179(7), 1347-1354.

Kimura, K., \& Kimura, A. (2014). A novel mechanism of microtubule length-dependent force to pull centrosomes toward the cell center. BioArchitecture, 1(2), 74-79.

Kiyomitsu, T. (2014). Mechanisms of daughter cell-size control during cell division. Trends Cell Biol.

Kiyomitsu, T., \& Cheeseman, I. M. (2013). Cortical dynein and asymmetric membrane elongation coordinately position the spindle in anaphase. Cell, 154(2), 391-402.

McNally, F. J. (2013). Mechanisms of spindle positioning. The Journal of Cell Biology, 200(2), 131-140.

Minc, N., Burgess, D., \& Chang, F. (2011). Influence of cell geometry on division-plane positioning. Cell, 144(3), 414-426.

Minc, N., \& Piel, M. (2012). Predicting division plane position and orientation. Trends Cell Biol, 22(4), 193-200.

Mitchison, T., Wühr, M., Nguyen, P., Ishihara, K., Groen, A., \& Field, C. M. (2012). Growth, interaction, and positioning of microtubule asters in extremely large vertebrate embryo cells. Cytoskeleton, 69(10), 738-750.

Stewart-Savage, J., \& Grey, R. D. (1982). The temporal and spatial relationships between cortical contraction, sperm trail formation, and pronuclear migration in fertilizedXenopus eggs. Wilhelm Roux's archives of developmental biology, 191(4), 241-245.

Tanimoto, H., Kimura, A., \& Minc, N. (2016). Shape-motion relationships of centering microtubule asters. The Journal of Cell Biology, 212(7), 777-787.

Wuhr, M., Tan, E. S., Parker, S. K., Detrich, H. W., 3rd, \& Mitchison, T. J. (2010). A model for cleavage plane determination in early amphibian and fish embryos. Curr Biol, 20(22), 2040-2045. 
6. Figure legends: 
(A)

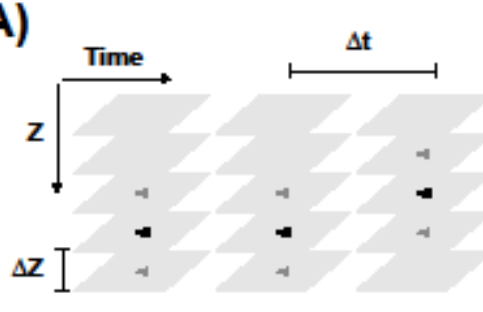

(C)

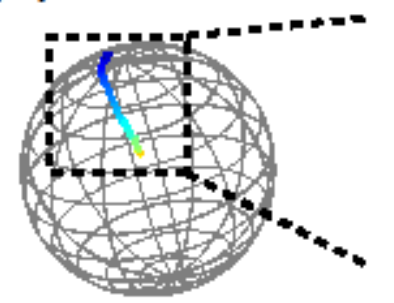

(D)

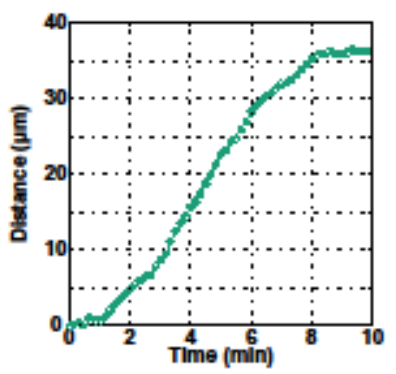

(B)

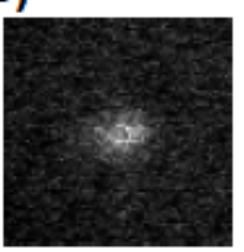

$+3 \mu \mathrm{m}$

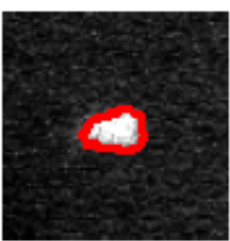

In Focus

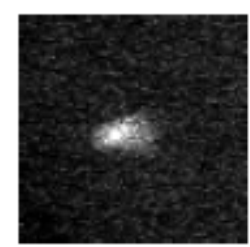

$-3 \mu \mathrm{m}$

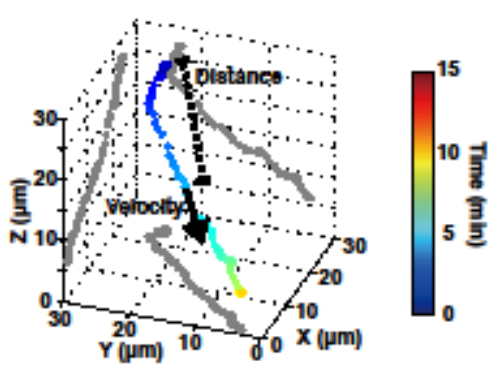

(E)

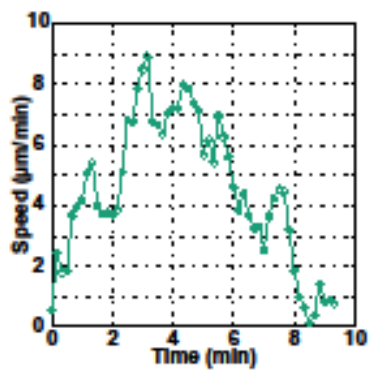

(F)

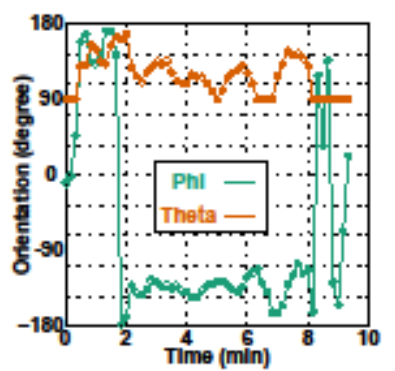

(G)
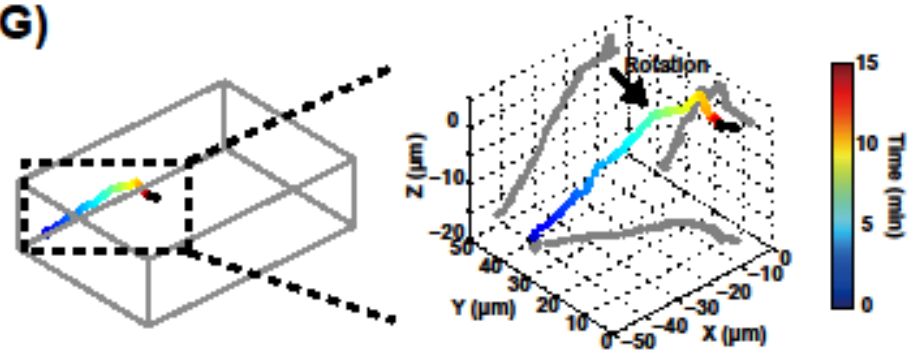

(H)

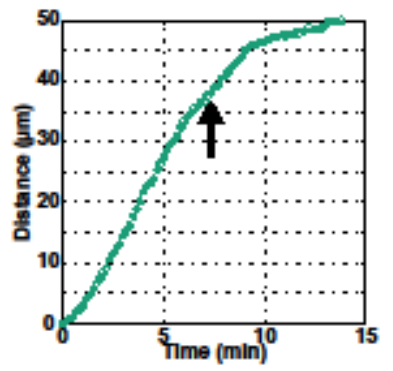

(I)

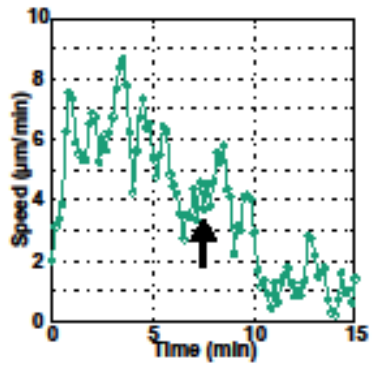

(K)

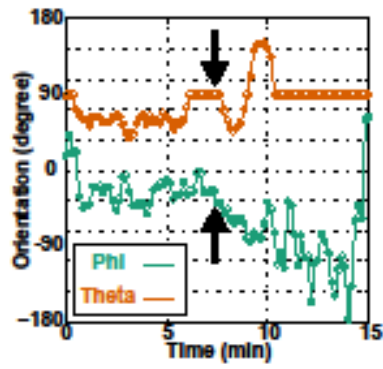

Figure 1. Tanimoto and Minc 


\section{Figure 1: Three-dimensional tracking of centering MT asters.}

(A) Sperm pronuclei at the aster center are imaged at each time point by acquiring confocal zslices. The slice interval $(\Delta \mathrm{Z})$ is set to be $3 \mu \mathrm{m}$ and 15 slices are acquired so that the $\mathrm{Z}$ stack covers the hemisphere of a sea urchin embryo whose radius is $45 \mu \mathrm{m}$. Each $\mathrm{Z}$ stack takes $\sim 8 \mathrm{sec}$ for the acquisition and the time interval $(\Delta \mathrm{t})$ is set to be $10 \mathrm{sec}$. (B) Confocal images of the sperm pronucleus visualized with a DNA dye Hoechst. Images in which the sperm pronucleus is in focus, and $3 \mu \mathrm{m}$ above/below are shown. The $\mathrm{Z}$ position of the center of the sperm aster is determined as the plane with maximum Hoechst signal. The XY position is determined as the center of mass of the 2D shape of the nucleus extracted by thresholding (red line). (C-F) Quantification of aster 3D centration. (C) 3D trajectory of a centering MT aster in a normal spherical cell. $X=Y=Z=0$ is the center of the egg (the same for $(G)$ ). The color indicates time. The trajectory consists from initial concaved penetration and long straight centration. The result shows that the precision of the tracking method is sufficient to confirm that the aster centration path is straight not only in XY but also in XZ and YZ. (D-F) Aster travelling distance (D), speed $(E)$, and velocity orientation $(F)$ are plotted as a function of time. See main text for definition.

(G-K) Non-straight aster centration in an egg shaped into a microfabricated chamber. The aster rotates during centration (indicated by a black arrow). Note that the tracking method identifies aster rotation in 3D, which is characterized by changes in both polar and azimuthal angles (K). 
(A)

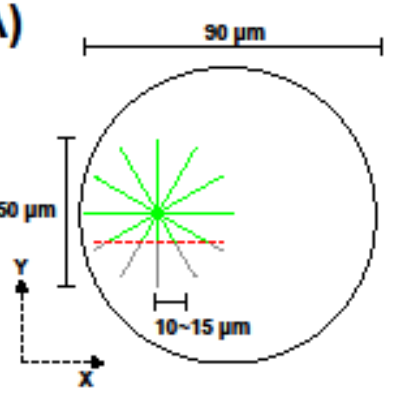

(B)

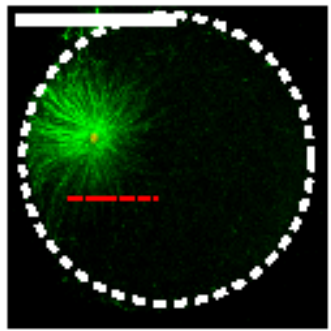

(C)

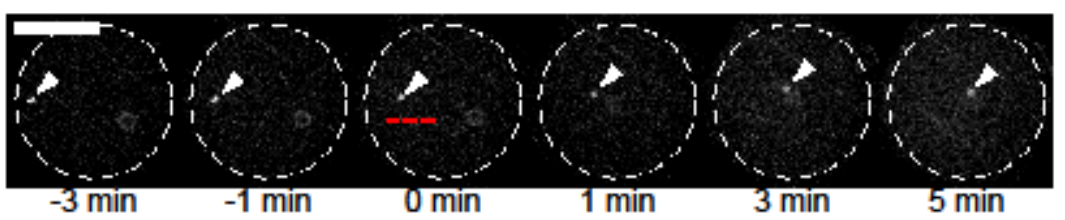

(D)

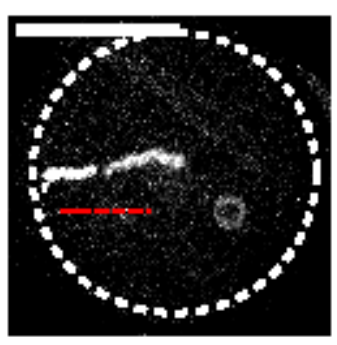

(E)

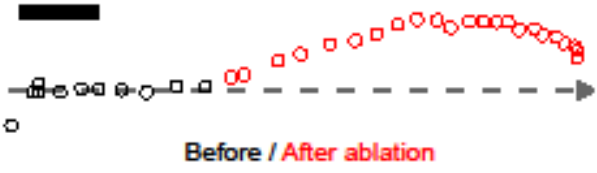

Figure 2. Tanimoto and Minc 


\section{Figure 2: Laser ablation assay for MT asters.}

(A) Schematic of the laser ablation assay. The setup for ablation at the aster side is shown. The red broken line indicates where the laser is applied (same for B-D) Intact and severed MTs are shown by green and gray colors, respectively. The ablation is performed in three $\mathrm{Z}$ heights: the aster focal plane and $5 \mu \mathrm{m}$ above/below. (B) Tubulin immunostaining of an ablated aster. An aster was fixed in situ immediately after side laser ablation. Both density and length of MTs at the ablation side are significantly reduced. (C-E) Aster side ablation. (C) Time-lapse confocal images of the male pronucleus during aster side laser ablation. Time 0 corresponds to the moment when laser ablation is done. Arrowhead: male pronucleus. (D) Time projection of fluorescent images shown in (C). (E) The position of the ablated aster is shown every $10 \mathrm{sec}$. The aster migrates from left to right, and black and red colors mark the aster center before and after ablation, respectively. The side ablation leads to an aster moving in a direction away from the MT ablation site, indicating the aster may be driven by outward forces. Bars: $50 \mu \mathrm{m}(\mathrm{B}-\mathrm{D})$ and $10 \mu \mathrm{m}(\mathrm{E})$. 